MSC 35L05

\title{
ВЕСОВОЕ ОДНОРОДНОЕ РАСПРЕДЕЛЕНИЕ И ЕГО ПРИЛОЖЕНИЯ
}

\section{Алзамили Х., Шишкина Э. Л.}

Белгородский государственный национальный исследовательский университет, Белгород, 308015, Россия

Воронежский Государственный Университет, Воронеж, 394018, Россия;

Белгородский государственный национальный исследовательский университет, Белгород, 308015, Россия

E-mail: alzamili.khitam@mail.ru, ilina_dico@mail.ru

Аннотация. Целью статьи является изучение связи между продолжением весового однородного распределения и весовым фундаментальным решением эллиптического оператора с операторами Бесселя, действующими по каждому аргументу. Эта задача для невесовых распределений рассматривалась Хёрмандером и наши результаты являются обобщением его результатов. Кроме того, рассмотрена задача Дирихле и получено равенство, дающее связь граничного условия и решения этой задачи Дирихле посредством $B$-потенциала Рисса.

Ключевые слова: весовое однородное распределение, оператор Бесселя, задача Дирихле, $B$-потенциал Рисса

Для цитирования: Алзамили Х., Шишкина Э. Л. 2021. Весовое однородное распределение и его приложения. Прикладная математика \& Физика. 53(4): 301-311. DOI 10.52575/2687-0959-2021-53-4-301-311.

\section{WEIGHTED HOMOGENEOUS DISTRIBUTION AND ITS APPLICATIONS}

\section{Khitam Alzamili, Elina Shishkina}

\author{
Belgorod State National Research University \\ Belgorod, 308015, Russia \\ Voronezh State University, \\ Voronezh, 394018, Russia; \\ Belgorod State National Research University, \\ Belgorod, 308015, Russia; \\ E-mail: alzamili.khitam@mail.ru, ilina_dico@mail.ru
}

Received December, 8, 2021

\begin{abstract}
The aim of the article is to study the relationship between the continuation of the weighted homogeneous distribution and the weighted fundamental solution of an elliptic operator with Bessel operators acting with respect to each argument. This problem for not weighted distribution was considered by Hörmander and our results are a generalization of his results. In addition, the Dirichlet problem is considered and an equality is obtained that gives a connection between the boundary condition and the solution of this Dirichlet problem by means of the Riesz $B$-potential.
\end{abstract}

Key words: weighted homogeneous distribution, Bessel operator, Dirichlet problem, Riesz B-potential .

For citation: Alzamili Khitam, Shishkina Elina. 2021. Weighted homogeneous distribution and its applications. Applied Mathematics \& Physics. 53(4): 301-311. (in Russian) DOI 10.52575/2687-0959-2021-53-4-301-311.

1. Введение и основные определения. В этом разделе приведены основные определения, включающие пространства основных функций, весовых обобщенных функций и обобщенной свертки.

Пусть $\mathbb{R}^{n}-n$-мерное евклидово пространство,

$$
\begin{aligned}
& \mathbb{R}_{+}^{n}=\left\{x=\left(x_{1}, \ldots, x_{n}\right) \in \mathbb{R}^{n}, \quad x_{1}>0, \ldots, x_{n}>0\right\}, \\
& \overline{\mathbb{R}}_{+}^{n}=\left\{x=\left(x_{1}, \ldots, x_{n}\right) \in \mathbb{R}^{n}, \quad x_{1} \geq 0, \ldots, x_{n} \geq 0\right\},
\end{aligned}
$$

$\gamma=\left(\gamma_{1}, \ldots, \gamma_{n}\right)-$ мультииндекс, состоящий из фиксированных положительных чисел $\gamma_{i}, i=1, \ldots, n$, и $|\gamma|=\gamma_{1}+$ $\ldots+\gamma_{n}$.

Рассмотрим открытое множество $\Omega$ в $\mathbb{R}^{n}$, симметричное относительно каждой гиперплоскости $x_{i}=0$, $i=1, \ldots, n$. Пусть $\Omega_{+}=\Omega \cap \mathbb{R}_{+}^{n}$ и $\bar{\Omega}_{+}=\Omega \cap \overline{\mathbb{R}}_{+}^{n}$. Имеем $\Omega_{+} \subseteq \mathbb{R}_{+}^{n}$ и $\bar{\Omega}_{+} \subseteq \overline{\mathbb{R}}_{+}^{n}$. Мы рассмотрим множество $C^{m}\left(\Omega_{+}\right)$, состоящее из $m$ раз дифференцируемых на оn $\Omega_{+}$функций. Через $C^{m}\left(\bar{\Omega}_{+}\right)$обозначим подмножество 
функций из $C^{m}\left(\Omega_{+}\right)$таких, что все производные этих функций по $x_{i}$ для любого $i=1, \ldots, n$ непрерывно продолжаются на $x_{i}=0$. Класс $C_{e v}^{m}\left(\bar{\Omega}_{+}\right)$состоит из функций $f \in C^{m}\left(\bar{\Omega}_{+}\right)$, таких, что $\left.\frac{\partial^{2 k+1} f}{\partial x_{i}^{2 k+1}}\right|_{x=0}=0$ для всех неотрицательных целых $k \leq m$ при $i=1, \ldots, n$ (см. [1] и [2], стр. 21 и далее). Пусть $C_{e v}^{\infty}\left(\bar{\Omega}_{+}\right)=\bigcap_{m=0}^{\infty} C_{e v}^{m}\left(\bar{\Omega}_{+}\right)$. Положим $C_{e v}^{\infty}\left(\overline{\mathbb{R}}_{+}^{n}\right)=C_{e v}^{\infty}$.

Пусть $\stackrel{\circ}{C}_{e v}^{\infty}\left(\bar{\Omega}_{+}\right)-$множество функций $f \in C_{e v}^{\infty}\left(\bar{\Omega}_{+}\right)$с компактным носителем.

Будем использовать обозначения $\stackrel{\circ}{C}_{e v}^{\infty}\left(\bar{\Omega}_{+}\right)=\mathcal{D}_{+}\left(\bar{\Omega}_{+}\right), \mathcal{D}_{+}\left(\overline{\mathbb{R}}_{+}^{n}\right)=\mathcal{D}_{+}$. Кроме $\mathcal{D}_{+}$будем также использовать множество $\mathscr{D}_{+}$функций, определенных также как и $\mathcal{D}_{+}$, но на $\mathbb{R}_{+}^{n}$.

Пространство $L_{p}^{\gamma}\left(\Omega_{+}\right), 1 \leq p<\infty$ состоит из измеримых на $\bar{\Omega}_{+}$функций, четных по каждой из своих переменных $x_{i}, i=1, \ldots, n$ таких, что если $f \in L_{p}^{\gamma}\left(\Omega_{+}\right)$, то

$$
\int_{\Omega_{+}}|f(x)|^{p} x^{\gamma} d x<\infty, \quad x^{\gamma}=\prod_{i=1}^{n} x_{i}^{\gamma_{i}}
$$

Будем использовать обозначения $L_{p}^{\gamma}=L_{p}^{\gamma}\left(\mathbb{R}_{+}^{n}\right)$ и

$$
\|f\|_{p, \gamma}=\left(\int_{\mathbb{R}_{+}^{n}}|f(x)|^{p} x^{\gamma} d x\right)^{1 / p} .
$$

Через $L_{p, l o c}^{\gamma}\left(\Omega_{+}\right)$будем обозначать множество функций $u$, определенных почти всюду на $\bar{\Omega}_{+}$, таких что $u \varphi \in L_{p}^{\gamma}\left(\Omega_{+}\right)$для всех $\varphi \in \mathcal{D}_{+}\left(\bar{\Omega}_{+}\right)$. Пусть $\mathcal{D}_{+}^{\prime}\left(\bar{\Omega}_{+}\right)-$сопряжённое пространство к $\mathcal{D}_{+}\left(\bar{\Omega}_{+}\right)$пространство. Каждой функции $u \in L_{1, l o c}^{\gamma}\left(\Omega_{+}\right)$сопоставляется регулярная весовая обобщенная функция $u \in \mathcal{D}_{+}^{\prime}\left(\bar{\Omega}_{+}\right)$, действующая по правилу

$$
(u, \varphi)_{\gamma}=\int_{\Omega_{+}} u(x) \varphi(x) x^{\gamma} d x, \quad \varphi \in \mathcal{D}_{+}\left(\bar{\Omega}_{+}\right) .
$$

Все остальные обобщенные функции $u \in \mathcal{D}_{+}^{\prime}\left(\bar{\Omega}_{+}\right)$будем называть сингулярными весовыми обобщенными $\oint$ фницями. Будем использовать обозначение $\mathcal{D}_{+}^{\prime}=\mathcal{D}_{+}^{\prime}\left(\overline{\mathbb{R}}_{+}^{n}\right)$. Аналогично определяется $\mathscr{D}_{+}^{\prime}$.

Например, сингулярной весовой обобщенной функцией является функция $\delta_{\gamma}$ :

$$
\left(\delta_{\gamma}, \varphi\right)_{\gamma}=\varphi(0), \quad \varphi \in \mathcal{D}_{+} .
$$

Для удобства будем также писать

$$
\left(\delta_{\gamma}, \varphi\right)_{\gamma}=\int_{\mathbb{R}_{+}^{n}} \delta_{\gamma}(x) \varphi(x) x^{\gamma} d x=\varphi(0)
$$

понимая такую запись как предел соответствующей последовательности.

Часть шара $|x| \leq r,|x|=\sqrt{x_{1}^{2}+\ldots+x_{n}^{2}}$, принадлежащую $\mathbb{R}_{+}^{n}$, будем обозначать $B_{r}^{+}(n)$. Граница $B_{r}^{+}(n)$ состоит из части сферы $S_{r}^{+}(n)=\left\{x \in \mathbb{R}_{+}^{n}:|x|=r\right\}$ и из частей координатных гиперплоскостей $x_{i}=0, i=1, \ldots, n$, таких что $|x| \leq r$.

Многомерный обобщенный сдвиг определяется равенством

$$
\left({ }^{\gamma} \mathbf{T}_{x}^{y} f\right)(x)={ }^{\gamma} \mathbf{T}_{x}^{y} f(x)=\left({ }^{\gamma_{1}} T_{x_{1}}^{y_{1}} \ldots{ }^{\gamma_{n}} T_{x_{n}}^{y_{n}} f\right)(x),
$$

где каждый одномерный обобщенный сдвиг ${ }^{\gamma_{i}} T_{x_{i}}^{y_{i}}$ acts for $i=1, \ldots, n$ имеет вид

$$
\begin{gathered}
\left({ }^{{ }^{\gamma_{i}}} T_{x_{i}}^{y_{i}} f\right)(x)=\frac{\Gamma\left(\frac{y_{i}+1}{2}\right)}{\sqrt{\pi} \Gamma\left(\frac{\gamma_{i}}{2}\right)} \times \\
\times \int_{0}^{\pi} f\left(x_{1}, \ldots, x_{i-1}, \sqrt{x_{i}^{2}+\tau_{i}^{2}-2 x_{i} y_{i} \cos \varphi_{i}}, x_{i+1}, \ldots, x_{n}\right) \sin ^{\gamma_{i}-1} \varphi_{i} d \varphi_{i} .
\end{gathered}
$$

Обобщенный сдвиг (2) подробно изучен в [3]. 
Обобщенная свертка, порожденная многомерным обобщенным сдвигом ${ }^{\gamma} \mathrm{T}_{x}^{y}$, определяется формулой

$$
(f * g)_{\gamma}(x)=(f * g)_{\gamma}=\int_{\mathbb{R}_{+}^{n}} f(y)\left({ }^{\gamma} \mathbf{T}_{x}^{y} g\right)(x) y^{\gamma} d y .
$$

2. Весовые однородные распределения. В этом разделе технику однородных распределений распространим на случай, когда присутствует степенной вес $x^{\gamma}$, что позволит применить полученные результаты к дифференциальным уравнениям с оператором Бесселя.

Пусть функция $u \in L_{1, l o c}^{\gamma}\left(\mathbb{R}_{+}^{n}\right)-$ однородная порядка $a$, то есть $u(t x)=t^{a} u(x)$ при $t>0$. Весовое распределение $u$ однородно порядка $a$ в $\mathbb{R}_{+}^{n}$, если выполняется следующее равенство

$$
(u, \varphi)_{\gamma}=t^{a}\left(u(x), \varphi_{t}(x)\right)_{\gamma}
$$

где $\varphi_{t}(x)=t^{n+|\gamma|} \varphi(t x), \varphi \in \mathscr{D}_{+}$. Если $u-$ весовое распределение в $\overline{\mathbb{R}}_{+}^{n}$ и (4) верно для всех $\varphi \in \mathcal{D}_{+}$, то $u-$ есть однородное распределение порядка $a$ в $\overline{\mathbb{R}}_{+}^{n}$.

Рассмотрим определение весового однородного распределения подробнее. Если функция $u \in L_{1, l o c}^{\gamma}\left(\mathbb{R}_{+}^{n}\right)$ однородна порядка $a$, то есть $u(t x)=t^{a} u(x)$ при $t>0$, то

$$
\begin{gathered}
(u(y), \varphi(y))_{\gamma}=\int_{\mathbb{R}_{+}^{n}} u(y) \varphi(y) y^{\gamma} d y=\{y=t x, t>0\}= \\
=t^{n+|\gamma|+a} \int_{\mathbb{R}_{+}^{n}} u(x) \varphi(t x) x^{\gamma} d x=t^{a}\left(u(x), \varphi_{t}(x)\right)_{\gamma},
\end{gathered}
$$

где $\varphi_{t}(x)=t^{n+|\gamma|} \varphi(t x), \varphi \in \mathscr{D}_{+}$. Обратно, из соотношения $(u(y), \varphi(y))_{\gamma}=t^{a}\left(u(x), \varphi_{t}(x)\right)_{\gamma}$ следует, что $u-$ однородна. Если $a>-n-|\gamma|$, то $u-$ интегрируема с весом $x^{\gamma}$ в некоторой окрестности нуля, поскольку при переходе к сферическим координатам $x=r \omega,|\omega|=1$ имеем $d x=r^{n+|\gamma|-1} \omega^{\gamma} d r d \omega$ и

$$
\begin{gathered}
\int_{U_{\varepsilon}(0)}|u(r \omega)| x^{\gamma} d x=\int_{0}^{\varepsilon} r^{a+n+|\gamma|-1} d r \int_{S_{1}^{+}(n)}|u(\omega)| \omega^{\gamma} d \omega= \\
=\frac{\varepsilon^{a+n+|\gamma|}}{a+n+|\gamma|} \int_{S_{1}^{+}(n)}|u(\omega)| \omega^{\gamma} d \omega<\infty .
\end{gathered}
$$

Теорема 2.1. Условия однородности весового однородного распределения и порядка а

$$
(u, \varphi)_{\gamma}=t^{a}\left(u, \varphi_{n, \gamma, t}\right)_{\gamma}, \quad \varphi_{n, \gamma, t}(x)=t^{n+|\gamma|} \varphi(t x), \quad \varphi \in \mathscr{D}_{+}
$$

$u$

$$
(u, \psi)_{\gamma}=0, \quad \psi \in \mathscr{D}_{+}, \quad \int_{0}^{\infty} r^{a+n+|\gamma|-1} \psi(r x) d r=0
$$

эквивалентны. Кроме того, для весовых однородных распределений и степени а справедлива формула

$$
\sum_{k=1}^{n} x_{k} \frac{\partial u}{\partial x_{k}}=a u
$$

Доказательство. Дифференцируя равенство (5) по $t$, получим

$$
\begin{gathered}
a t^{a-1}\left(u(x), t^{n+|\gamma|} \varphi(t x)\right)_{\gamma}+ \\
+t^{a}\left(u(x),(n+|\gamma|) t^{n+|\gamma|-1} \varphi(t x)+t^{n+|\gamma|-1} \frac{d \varphi(t x)}{d t}\right)_{\gamma}=0 .
\end{gathered}
$$

Поскольку

$$
\frac{d \varphi(t x)}{d t}=\sum_{k=1}^{n} \frac{\partial \varphi(t x)}{\partial x_{k}} \frac{d\left(t x_{k}\right)}{d t}=\sum_{k=1}^{n} x_{k} \frac{\partial \varphi(t x)}{\partial x_{k}},
$$

то равенство (8) может быть записано в виде

$$
a t^{a-1}\left(u(x), t^{n+|\gamma|} \varphi(t x)\right)_{\gamma}+
$$




$$
+t^{a}\left(u(x),(n+|\gamma|) t^{n+|\gamma|-1} \varphi(t x)+t^{n+|\gamma|-1} \sum_{k=1}^{n} x_{k} \frac{\partial \varphi(t x)}{\partial x_{k}}\right)_{\gamma}=0 .
$$

Положив $t=1$ в (9), будем иметь

$$
(a+n+|\gamma|)(u, \varphi)_{\gamma}+(u, \lambda \varphi)_{\gamma}=0
$$

где $\lambda=\sum_{k=1}^{n} x_{k} \frac{\partial}{\partial x_{k}}$.

Рассмотрим уравнение

$$
(a+n+|\gamma|) \varphi(x)+\sum_{k=1}^{n} x_{k} \frac{\partial}{\partial x_{k}} \varphi(x)=\psi(x)
$$

и покажем, что у него есть решение в $\mathscr{D}_{+}$. Переходя в $(11)$ к сферическим координатам $x=r \omega$, получим

$$
\frac{\partial}{\partial r}\left(r^{a+n+|\gamma|} \varphi(r \omega)\right)=\psi(r \omega) r^{a+n+|\gamma|-1}
$$

Действительно,

$$
\begin{gathered}
\frac{\partial}{\partial r}\left(r^{a+n+|\gamma|} \varphi(r \omega)\right)=(a+n+|\gamma|) r^{a+n+|\gamma|-1} \varphi(r \omega)+r^{a+n+|\gamma|} \frac{\partial \varphi(r \omega)}{\partial r}= \\
=(a+n+|\gamma|) r^{a+n+|\gamma|-1} \varphi(r \omega)+r^{a+n+|\gamma|} \sum_{k=1}^{n} \frac{\partial \varphi(r \omega)}{\partial\left(r \omega_{k}\right)} \frac{\partial\left(r \omega_{k}\right)}{\partial r}= \\
=(a+n+|\gamma|) r^{a+n+|\gamma|-1} \varphi(r \omega)+r^{a+n+|\gamma|-1} \sum_{k=1}^{n} r \omega_{k} \frac{\partial \varphi(r \omega)}{\partial\left(r \omega_{k}\right)}= \\
=r^{a+n+|\gamma|-1}\left[(a+n+|\gamma|) \varphi(x)+\sum_{k=1}^{n} x_{k} \frac{\partial}{\partial x_{k}} \varphi(x)\right]=r^{a+n+|\gamma|-1} \psi(x) .
\end{gathered}
$$

Следовательно,

$$
\psi=(a+n+|\gamma|) \varphi+\lambda \varphi
$$

равенство

$$
\int_{0}^{\infty} r^{a+n+|\gamma|-1} \psi(r x) d r=\int_{0}^{\infty} \frac{\partial}{\partial r}\left(r^{a+n+|\gamma|} \varphi(r \omega)\right) d r=\left.r^{a+n+|\gamma|} \varphi(r \omega)\right|_{0} ^{\infty}=0
$$

верно и $\psi \in \mathscr{D}_{+}$. Таким образом, из (12) и (10) следует равенство (6).

Теперь докажем (7). Для $(u, \lambda \varphi)_{\gamma}$ получим

$$
\begin{gathered}
(u, \lambda \varphi)_{\gamma}=\left(u(x), \sum_{k=1}^{n} x_{k} \frac{\partial \varphi(x)}{\partial x_{k}}\right)_{\gamma}=\sum_{k=1}^{n} \int_{\mathbb{R}_{+}^{n}} u(x) x_{k} \frac{\partial \varphi(x)}{\partial x_{k}} x^{\gamma} d x= \\
=\sum_{k=1}^{n} \int_{\mathbb{R}_{+}^{n-1}} x_{1}^{\gamma_{1}} \ldots x_{k-1}^{\gamma_{k-1}} x_{k+1}^{\gamma_{k+1}} \ldots x_{n}^{\gamma_{n}} d x_{1} \ldots d x_{k-1} d x_{k+1} \ldots d x_{n}\left[\int_{0}^{\infty} u(x) \frac{\partial \varphi(x)}{\partial x_{k}} x_{k}^{\gamma_{k}+1} d x_{k}\right] .
\end{gathered}
$$

Применим формулу интегрирования по частям к интегралу по $x_{k}$ :

$$
\begin{gathered}
\int_{0}^{\infty} u(x) \frac{\partial \varphi(x)}{\partial x_{k}} x_{k}^{\gamma_{k}+1} d x_{k}=\left\{U=u(x) x_{k}^{\gamma_{k}+1}, d V=\frac{\partial \varphi(x)}{\partial x_{k}} d x_{k}\right\}= \\
=\left.u(x) \varphi(x) x_{k}^{\gamma_{k}+1}\right|_{0} ^{\infty}-\int_{0}^{\infty}\left[x_{k} \frac{\partial u}{\partial x_{k}}+\left(1+\gamma_{k}\right) u(x)\right] \varphi(x) x_{k}^{\gamma_{k}} d x_{k}= \\
=-\int_{0}^{\infty} x_{k} \frac{\partial u}{\partial x_{k}} \varphi(x) x_{k}^{\gamma_{k}} d x_{k}-\left(1+\gamma_{k}\right) \int_{0}^{\infty} u(x) \varphi(x) x_{k}^{\gamma_{k}} d x_{k} .
\end{gathered}
$$

Суммируя по $k$ от 1 до $n$ и возвращаясь к интегралу (13), будем иметь

$$
(u, \lambda \varphi)_{\gamma}=-(\lambda, \varphi)_{\gamma}-(n+|\gamma|)(u, \varphi)_{\gamma}
$$


Подставляя (14) в (10), получим

$$
(a+n+|\gamma|)(u(x), \varphi(x))_{\gamma}-\left(\sum_{k=1}^{n} x_{k} \frac{\partial u}{\partial x_{k}}, \varphi(x)\right)_{\gamma}-(n+|\gamma|)(u(x), \varphi(x))_{\gamma}=0
$$

или

$$
a(u(x), \varphi(x))_{\gamma}=\left(\sum_{k=1}^{n} x_{k} \frac{\partial u}{\partial x_{k}}, \varphi(x)\right)_{\gamma}
$$

что дает (7).

3. Продолжение весовых однородных распределений. Продолжение весового однородного распределения с $\mathbb{R}_{+}^{n}$ до распределения на $\overline{\mathbb{R}}_{+}^{n}$ является нетривиальной задачей, но необходимой для построения фундаментальных решений. В этом разделе мы построим продолжение весового однородного распределения порядка $a$.

Теорема 3.1. Пусть $u \in \mathscr{D}_{+}^{\prime}-$ весовое однородное распределение порядка а. Если $a \neq k, k \in \mathbb{Z}, k \leq-n-|\gamma|$, то распределение и имеет единственное продолжение $u^{*} \in \mathcal{D}_{+}^{\prime}$ однородное порядка $а$. Если $а \neq 1-n-|\gamma|$, то $\left(B_{\gamma_{j}} u\right)^{*}=B_{\gamma_{j}} u^{*}, B_{\gamma_{j}}=\frac{\partial^{2}}{\partial x_{j}^{2}}+\frac{\gamma_{j}}{x_{j}} \frac{\partial}{\partial x_{j}}$. Отображение $u \rightarrow u^{*}$ непрерьвно.

Доказательство. Сначала докажем существование весового распределения $u^{*} \in \mathcal{D}_{+}^{\prime}$,однородного порядка $a$, которое является продолжением $u \in \mathscr{D}_{+}^{\prime}$.

Если $u-$ функция и $\varphi \in \mathscr{D}_{+}$, то переходя к сферическим координатам $x=r \omega$, получим

$$
(u, \varphi)_{\gamma}=\int_{\mathbb{R}_{+}^{n}} u(x) \varphi(x) x^{\gamma} d x=\int_{0}^{\infty} \int_{S_{1}^{+}(n)} u(\omega) \varphi(r \omega) r^{a+n+|\gamma|-1} \omega^{\gamma} d r d \omega .
$$

На основе этого равенства введем одномерное распределение (не весовое)

$$
\left(R_{a} \varphi\right)(x)=\left(t_{+}^{a+n+|\gamma|-1}, \varphi(t x)\right), \quad \varphi \in \stackrel{\circ}{C}_{e v}^{\infty}\left(\overline{\mathbb{R}}_{+}^{n}\right) .
$$

Функция $R_{a} \varphi$ однородна порядка $-n-|\gamma|-a$, то есть $\left(R_{a} \varphi\right)(b x)=b^{-n-|\gamma|-a}\left(R_{a} \varphi\right)(x)$. Действительно,

$$
\begin{gathered}
\left(R_{a} \varphi\right)(b x)=\left(t_{+}^{a+n+|\gamma|-1}, \varphi(b t x)\right)=\int_{0}^{\infty} t^{a+n+|\gamma|-1} \varphi(b t x) d t=\{b t=y\}= \\
=b^{-n-|\gamma|-a} \int_{0}^{\infty} y^{a+n+|\gamma|-1} \varphi(y x) d y=b^{-n-|\gamma|-a}\left(R_{a} \varphi\right)(x) .
\end{gathered}
$$

Из [6] следует, что $R_{a}$ есть непрерывное отображение из $\stackrel{\circ}{C}_{e v}^{\infty}(K)$ в $\stackrel{\circ}{C}_{e v}^{\infty}\left(\mathbb{R}_{+}^{n}\right)$ для любого компакта $K \subset \mathbb{R}_{+}^{n}$. Выберем фиксированную функцию $\psi \in \mathscr{D}_{+}$, такую что

$$
\int_{0}^{\infty} \psi(t x) \frac{d t}{t}=1, \quad x \neq 0 .
$$

Тогда $\psi R_{a} \varphi \in \mathscr{D}_{+}$и

$$
\begin{gathered}
R_{a}\left(\psi R_{a} \varphi\right)(x)=\int_{0}^{\infty} t^{a+n+|\gamma|-1} \psi(t x)\left(R_{a} \varphi\right)(t x) d t= \\
=\left(R_{a} \varphi\right)(x) \int_{0}^{\infty} \psi(t x) \frac{d t}{t}=\left(R_{a} \varphi\right)(x) .
\end{gathered}
$$

Таким образом, $u\left(\psi R_{a} \varphi\right)$ всегда не зависит от $\psi$ и $u\left(\psi R_{a} \varphi\right)=u(\varphi)$, если $\varphi \in \mathscr{D}_{+}$. Следовательно,

$$
\left(u^{*}, \varphi\right)_{\gamma}=\left(u, \psi R_{a} \varphi\right)_{\gamma}, \quad \varphi \in \mathcal{D}_{+}
$$

определяет распределение $u^{*}$ в $\overline{\mathbb{R}}_{+}^{n}$, продолжающее $u$. Отображение $u \rightarrow u^{*}$ непрерывно. Поскольку

$$
\left(R_{a} \varphi_{t}\right)(x)=\left(r_{+}^{a+n+|\gamma|-1}, t^{n+|\gamma|} \varphi(r t x)\right)=t^{-a} R_{a} \varphi(x), \quad \varphi_{t}(x)=t^{n+|\gamma|} \varphi(t x),
$$


что дает однородность $u^{*}$. Наконец заметим, что $\left(B_{\gamma_{j}} u\right)^{*}-B_{\gamma_{j}} u^{*}$ однородно порядка $a-2$ и имеет носитель в нуле 0 , таким образом, должно равняться нулю. Это завершает доказательство.

4. Весовое фундаментальное решение $B$-эллиптического уравнения Цель этого раздела показать, как теорию весовых однородных распределений можно использовать для получения того, что мы называем весовыми фундаментальными решениями уравнений в частных производных.

Распределение $E \in \mathcal{D}_{+}^{\prime}$ называется весовым фундаментальным решением дифференциального оператора $L=\sum_{i=1}^{m} a_{i} B_{\gamma_{i}}$ с операторами Бесселя, с постоянными (комплексными) коэффициентами, если $L E=\delta_{\gamma}$.

Весовое фундаментальное решение эллиптического оператора с оператором Бесселя, действующим по одной переменной, было получено И. А. Киприяновым в [2] другим методом.

Теорема 4.1. Пусть $u_{1}, \ldots, u_{n} \in \mathcal{D}_{+}^{\prime}$ функции, однородные порядка $2-n-|\gamma|$ в $\mathbb{R}_{+}^{n}$, удовлетворяющие условию $\sum_{j=1}^{n} B_{\gamma_{j}} u_{j}=0$. Тогда $\sum_{j=1}^{n} B_{\gamma_{j}} u_{j}^{*}=c \delta_{\gamma}$, где $c-$ некоторая константа.

Доказательство. В силу теоремы 3.1 , весовое распределение $\sum_{j=1}^{n} B_{\gamma_{j}} u_{j}^{*}$ однородно порядка $-n-|\gamma|$ и имеет носитель в 0 , следовательно $\sum_{j=1}^{n} B_{\gamma_{j}} u_{j}^{*}=c \delta_{\gamma}$ для некоторой константы $c$.

Лемма 4.1. Пусть $x \in \mathbb{R}_{+}^{n}, n>1 u$

$$
E(x)= \begin{cases}\frac{1}{\left|S_{1}^{+}(n)\right|_{\gamma}} \ln |x|, & n+|\gamma|=2 ; \\ \frac{|x|^{2-n-|\gamma|}}{(2-n-|\gamma|)\left|S_{1}^{+}(n)\right|_{\gamma}}, & n+|\gamma|>2,\end{cases}
$$

где

$$
\left|S_{1}^{+}(n)\right|_{\gamma}=\int_{S_{1}^{+}(n)} x^{\gamma} d S=\frac{\prod_{i=1}^{n} \Gamma\left(\frac{\gamma_{i}+1}{2}\right)}{2^{n-1} \Gamma\left(\frac{n+|\gamma|}{2}\right)}
$$

то для $|x|>\varepsilon \forall \varepsilon>0$ имеем

$$
\triangle_{\gamma} E(x)=0
$$

Доказательство. Рассмотрим сначала случай $n+|\gamma|>2$. Получим

$$
\begin{aligned}
& \triangle_{\gamma} E(x)=\sum_{j=1}^{n} B_{\gamma_{j}} E(x)=\sum_{j=1}^{n} \frac{1}{x_{j}^{\gamma_{j}}} \frac{\partial}{\partial x_{j}} x_{j}^{\gamma_{j}} \frac{\partial}{\partial x_{j}} E(x)= \\
& =\frac{1}{(2-n-|\gamma|)\left|S_{n}^{+}\right|_{\gamma}} \sum_{j=1}^{n} \frac{1}{x_{j}^{\gamma_{j}}} \frac{\partial}{\partial x_{j}} x_{j}^{\gamma_{j}} \frac{\partial}{\partial x_{j}}|x|^{2-n-|\gamma|}= \\
& =\frac{1}{(2-n-|\gamma|)\left|S_{n}^{+}\right|_{\gamma}} \sum_{j=1}^{n} \frac{1}{x_{j}^{\gamma_{j}}} \frac{\partial}{\partial x_{j}} x_{j}^{\gamma_{j}} \frac{(2-n-|\gamma|)}{2}|x|^{-n-|\gamma|} 2 x_{j}= \\
& =\frac{1}{\left|S_{n}^{+}\right|_{\gamma}} \sum_{j=1}^{n} \frac{1}{x_{j}^{\gamma_{j}}} \frac{\partial}{\partial x_{j}}|x|^{-n-|\gamma|} x_{j}^{1+\gamma_{j}}= \\
& =\frac{1}{\left|S_{n}^{+}\right|_{\gamma}} \sum_{j=1}^{n} \frac{1}{x_{j}^{\gamma_{j}}}\left[\frac{(-n-|\gamma|)}{2}|x|^{-n-|\gamma|-2} 2 x_{j}^{2+\gamma_{j}}+\left(1+\gamma_{j}\right)|x|^{-n-|\gamma|} x_{j}^{\gamma_{j}}\right]= \\
& =\frac{1}{\left|S_{n}^{+}\right|_{\gamma}} \sum_{j=1}^{n}\left[(-n-|\gamma|)|x|^{-n-|\gamma|-2} x_{j}^{2}+\left(1+\gamma_{j}\right)|x|^{-n-|\gamma|}\right]= \\
& =\frac{1}{\left|S_{n}^{+}\right|_{\gamma}}\left[(-n-|\gamma|)|x|^{-n-|\gamma|}+(n+|\gamma|)|x|^{-n-|\gamma|}\right]=0 .
\end{aligned}
$$

Теперь рассмотрим случай $n+|\gamma|=2$ :

$$
\begin{gathered}
\triangle_{\gamma} E(x)=\sum_{j=1}^{n} B_{\gamma_{j}} E(x)=\sum_{j=1}^{n} \frac{1}{x_{j}^{\gamma_{j}}} \frac{\partial}{\partial x_{j}} x_{j}^{\gamma_{j}} \frac{\partial}{\partial x_{j}} E(x)= \\
=\frac{1}{\left|S_{n}^{+}\right|_{\gamma}} \sum_{j=1}^{n} \frac{1}{x_{j}^{\gamma_{j}}} \frac{\partial}{\partial x_{j}} x_{j}^{\gamma_{j}} \frac{\partial}{\partial x_{j}} \ln |x|=\frac{1}{\left|S_{n}^{+}\right|_{\gamma}} \sum_{j=1}^{n} \frac{1}{x_{j}^{\gamma_{j}}} \frac{\partial}{\partial x_{j}}|x|^{-2} x_{j}^{1+\gamma_{j}}=
\end{gathered}
$$




$$
\begin{gathered}
=\frac{1}{\left|S_{n}^{+}\right|_{\gamma}} \sum_{j=1}^{n} \frac{1}{x_{j}^{\gamma_{j}}}\left[-2|x|^{-4} x_{j}^{2+\gamma_{j}}+\left(1+\gamma_{j}\right)|x|^{-2} x_{j}^{\gamma_{j}}\right]= \\
=\frac{1}{\left|S_{n}^{+}\right|_{\gamma}} \sum_{j=1}^{n}\left[-2|x|^{-4} x_{j}^{2}+\left(1+\gamma_{j}\right)|x|^{-2}\right]= \\
=\frac{1}{\left|S_{n}^{+}\right|_{\gamma}}\left[-2|x|^{-2}+(n+|\gamma|)|x|^{-2}\right]=0,
\end{gathered}
$$

так как $n+|\gamma|=2$.

Теорема 4.2. Пусть $x \in \mathbb{R}_{n}^{+}, n>1 u$

$$
E(x)= \begin{cases}\frac{1}{\left|S_{2}^{+}\right|_{\gamma} \ln |x|,}, & n+|\gamma|=2 ; \\ \frac{|x|^{2-n-|\gamma|}}{(2-n-|\gamma|)\left|S_{n}^{+}\right|_{\gamma},} & n+|\gamma|>2,\end{cases}
$$

$\operatorname{mozдa~} B_{\gamma_{j}} E \in L_{l o c, \gamma}^{1}\left(\mathbb{R}_{+}^{n}\right) u$

$$
\triangle_{\gamma} E=\delta_{\gamma}
$$

Доказательство. Докажем сначала, что $B_{\gamma_{j}} E \in L_{l o c, \gamma}^{1}\left(\mathbb{R}_{+}^{n}\right)$. Для $\varphi \in \stackrel{\circ}{C}_{e v}^{\infty}\left(\overline{\mathbb{R}}_{+}^{n}\right)$ имеем

$$
\begin{aligned}
& \left(B_{\gamma_{j}} E, \varphi\right)_{\gamma}=\int_{\mathbb{R}_{+}^{n}} B_{\gamma_{j}} E(x) \varphi(x) x^{\gamma} d x=\int_{\mathbb{R}_{+}^{n}}\left(\frac{1}{x_{j}^{\gamma_{j}}} \frac{\partial}{\partial x_{j}} x_{j}^{\gamma_{j}} \frac{\partial}{\partial x_{j}} E(x)\right) \varphi(x) x^{\gamma} d x= \\
= & \int_{\mathbb{R}_{+}^{n-1}} x_{1}^{\gamma_{1}} \ldots x_{j-1}^{\gamma_{j-1}} x_{j+1}^{\gamma_{j+1}} \ldots x_{n}^{\gamma_{n}} d x_{1} \ldots d x_{j-1} d x_{j+1} \ldots d x_{n} \int_{0}^{\infty}\left(\frac{\partial}{\partial x_{j}} x_{j}^{\gamma_{j}} \frac{\partial}{\partial x_{j}} E(x)\right) \varphi(x) d x_{j} .
\end{aligned}
$$

Интегрируя по частям по $x_{j}$, получим

$$
\begin{gathered}
\int_{0}^{\infty}\left(B_{\gamma_{j}} E(x)\right) \varphi(x) x_{j}^{\gamma_{j}} d x_{j}=\int_{0}^{\infty}\left(\frac{1}{x_{j}^{\gamma_{j}}} \frac{\partial}{\partial x_{j}} x_{j}^{\gamma_{j}} \frac{\partial}{\partial x_{j}} E(x)\right) \varphi(x) x_{j}^{\gamma_{j}} d x_{j}= \\
=\int_{0}^{\infty}\left(\frac{\partial}{\partial x_{j}} x_{j}^{\gamma_{j}} \frac{\partial}{\partial x_{j}} E(x)\right) \varphi(x) d x_{j}=\left\{U=\varphi(x), d V=\frac{\partial}{\partial x_{j}} x_{j}^{\gamma_{j}} \frac{\partial}{\partial x_{j}} E(x) d x_{j}\right\}= \\
=\left.x_{j}^{\gamma_{j}} \frac{\partial}{\partial x_{j}} E(x) \varphi(x)\right|_{0} ^{\infty}-\int_{0}^{\infty} x_{j}^{\gamma_{j}}\left(\frac{\partial}{\partial x_{j}} E(x)\right) \frac{\partial}{\partial x_{j}} \varphi(x) d x_{j}= \\
=-\int_{0}^{\infty}\left(\frac{\partial}{\partial x_{j}} E(x)\right) x_{j}^{\gamma_{j}} \frac{\partial}{\partial x_{j}} \varphi(x) d x_{j}= \\
=\left\{U=x_{j}^{\gamma_{j}} \frac{\partial}{\partial x_{j}} \varphi(x), d V=\frac{\partial}{\partial x_{j}} E(x) d x_{j}\right\}= \\
=\left.\int_{0}^{\gamma_{j}} \frac{\partial}{\partial x_{j}} \varphi(x) E(x)\right|_{0} ^{\infty}+\int_{0}^{\infty}\left(\frac{\partial}{\partial x_{j}} x_{j}^{\gamma_{j}} \frac{\partial}{\partial x_{j}} \varphi(x)\right) E(x) d x_{j}= \\
\left.\frac{x_{j}}{\partial x_{j}} x_{j}^{\gamma_{j}} \frac{\partial}{\partial x_{j}} \varphi(x)\right) E(x) x_{j}^{\gamma_{j}} d x_{j}=\int_{0}^{\infty}\left(B_{\gamma_{j}} \varphi(x)\right) E(x) x_{j}^{\gamma_{j}} d x_{j} .
\end{gathered}
$$

Следовательно,

$$
\begin{aligned}
& \left(B_{\gamma_{j}} E, \varphi\right)_{\gamma}=\left(E, B_{\gamma_{j}} \varphi\right)_{\gamma}=\lim _{\varepsilon \rightarrow 0} \int_{\{|x|>\varepsilon\}^{+}}\left(B_{\gamma_{j}} \varphi(x)\right) E(x) x^{\gamma} d x= \\
= & \lim _{\varepsilon \rightarrow 0}\left[\int_{\mathbb{R}_{+}^{n}}\left(B_{\gamma_{j}} \varphi(x)\right) E(x) x^{\gamma} d x-\int_{\{|x| \leq \varepsilon\}^{+}}\left(B_{\gamma_{j}} \varphi(x)\right) E(x) x^{\gamma} d x\right] .
\end{aligned}
$$


Используя формулу (4) из [7], получим

$$
\left(B_{\gamma_{j}} E, \varphi\right)_{\gamma}=\int_{\mathbb{R}_{n}^{+}}\left(B_{\gamma_{j}} \varphi(x)\right) E(x) x^{\gamma} d x-\lim _{\varepsilon \rightarrow 0} \int_{\{|x|=\varepsilon\}^{+}} \frac{\partial \varphi(x)}{\partial x_{j}} E(x) \cos \left(\vec{v}, \vec{e}_{j}\right) x^{\gamma} d S,
$$

где $\vec{v}$ - направление внешней нормали к границе $\{|x|=\varepsilon\}^{+}, \vec{e}_{j}-$ направление оси $O x_{j}$. Поскольку $\cos \left(\vec{v}, \vec{e}_{j}\right)=\frac{x_{j}}{|x|}$, то

$$
\left(B_{\gamma_{j}} E, \varphi\right)_{\gamma}=\int_{\mathbb{R}_{+}^{n}}\left(B_{\gamma_{j}} \varphi(x)\right) E(x) x^{\gamma} d x-\lim _{\varepsilon \rightarrow 0} \int_{\{|x|=\varepsilon\}^{+}} \frac{\partial \varphi(x)}{\partial x_{j}} E(x) \frac{x_{j}}{|x|} x^{\gamma} d S .
$$

Имеем

$$
\lim _{\varepsilon \rightarrow 0} \int_{\{|x|=\varepsilon\}^{+}} \frac{\partial \varphi(x)}{\partial x_{j}} E(x) \frac{x_{j}}{|x|} x^{\gamma} d S=0
$$

и, следовательно,

$$
\left(B_{\gamma_{j}} E, \varphi\right)_{\gamma}=\int_{\mathbb{R}_{+}^{n}}\left(B_{\gamma_{j}} E(x)\right) \varphi(x) x^{\gamma} d x
$$

то есть, весовое распределение $B_{\gamma_{j}} E$ определяемое функцией $B_{\gamma_{j}} E(x)$ локально интегрируемо с весом $x^{\gamma}$.

Поскольку для $|x|>\varepsilon, \forall \varepsilon>0$ выполняется равенство

$$
\triangle_{\gamma} E(x)=0
$$

то снова используя формулу (4) из [7], будем иметь

$$
\begin{aligned}
& \left(\Delta_{\gamma} E, \varphi\right)_{\gamma}=\left(E, \Delta_{\gamma} \varphi\right)_{\gamma}=\lim _{\varepsilon \rightarrow 0} \int_{\{|x|>\varepsilon\}^{+}} E(x)\left(\triangle_{\gamma} \varphi(x)\right) x^{\gamma} d x= \\
& =\lim _{\varepsilon \rightarrow 0} \int_{\{|x|>\varepsilon\}^{+}}\left[E(x)\left(\triangle_{\gamma} \varphi(x)\right)-\left(\triangle_{\gamma} E(x)\right) \varphi(x)\right] x^{\gamma} d x= \\
& =\lim _{\varepsilon \rightarrow 0} \int_{\{|x|=\varepsilon\}^{+}}\left(E(x) \frac{\partial \varphi(x)}{\partial \vec{v}}-\varphi(x) \frac{\partial E(x)}{\partial \vec{v}}\right) x^{\gamma} d S=\varphi(0) .
\end{aligned}
$$

Доказательство закончено.

5. Задача Дирихле. Основным назначением весового фундаментального решения является то, что оно позволяет решать неоднородные уравнения или задачу Дирихле с неоднородным условием, в которых неоднородный член имеет компактный носитель.

В этом пункте строится весовое фундаментальное решение задачи Дирихле, т. е. решением задачи Дирихле с весовой дельта-функцией в граничном условии. Решение задачи Дирихле с произвольной функцией в граничном условии в этом случае можно записать как обобщенную свертку (3) функции с весовым фундаментальным решением задачи Дирихле, если эта обобщенная свертка существует.

Пусть $\Delta_{\gamma, a}=\Delta_{\gamma}+B_{a}$. В этом пункте рассмотрим задачу Дирихле

$$
\begin{gathered}
\Delta_{\gamma, a} u=0, \quad u=u(x, y), \\
u(x, 0)=f(x) .
\end{gathered}
$$

Пусть $\vec{e}=\left(e_{1}, \ldots, e_{n}\right)$ является ортонормированным базисом в $\mathbb{R}^{n}, \nabla_{\gamma, a}^{\prime}=\left(\frac{1}{x_{1}^{\gamma_{1}}} \frac{\partial}{\partial x_{1}}, \ldots, \frac{1}{x_{n}^{\gamma_{n}}} \frac{\partial}{\partial x_{n}}, \frac{1}{y^{a}} \frac{\partial}{\partial y}\right)-$ первый взвешенный оператор набла, $\nabla_{\gamma, a}^{\prime \prime}=\left(x_{1}^{\gamma_{1}} \frac{\partial}{\partial x_{1}}, \ldots, x_{n}^{\gamma_{n}} \frac{\partial}{\partial x_{n}}, y^{a} \frac{\partial}{\partial y}\right)-$ второй взвешенный оператор набла, тогда $\left(\nabla_{\gamma, a}^{\prime} \cdot \nabla_{\gamma, a}^{\prime \prime}\right)=\Delta_{\gamma, a}$. Если $\vec{F}=\vec{F}(x, y)=\left(F_{1}(x, y), \ldots, F_{n+1}(x, y)\right)$ - векторное поле, то $d i v_{\gamma, a}^{\prime} \vec{F}=$ $\left(\nabla_{\gamma, a}^{\prime} \cdot \vec{F}\right)=\frac{1}{x_{1}^{\gamma_{1}}} \frac{\partial F_{1}}{\partial x_{1}}+\ldots+\frac{1}{x_{i}^{\gamma_{n}}} \frac{\partial F_{n}}{\partial x_{n}}+\frac{1}{y^{a}} \frac{\partial F_{n+1}}{\partial y}-$ первая весовая дивергенция, $\operatorname{div}_{\gamma, a}^{\prime \prime} \vec{F}=\left(\nabla_{\gamma, a}^{\prime \prime} \cdot \vec{F}\right)=x_{1}^{\gamma_{1}} \frac{\partial F_{1}}{\partial x_{1}}+$ $\ldots+x_{n}^{\gamma_{n}} \frac{\partial F_{n}}{\partial x_{n}}+y^{a} \frac{\partial F_{n+1}}{\partial y}-$ вторая весовая дивергенция.

Уравнение (16) можно переписать в виде $d i v_{\gamma, a}^{\prime}\left(\nabla_{\gamma, a}^{\prime \prime} u\right)=0$. 
Введя новую переменную $\eta=\left(\frac{y}{1-a}\right)^{1-a}$, получим

$$
\begin{gathered}
\frac{\partial \eta}{\partial y}=\left(\frac{y}{1-a}\right)^{-a}, \\
\frac{\partial^{2} u}{\partial y^{2}}+\frac{a}{y} \frac{\partial u}{\partial y}=\left(\frac{y}{1-a}\right)^{-2 a} u_{\eta \eta}=\eta^{\frac{2 a}{a-1}} u_{\eta \eta}
\end{gathered}
$$

и уравнение (16) перепишется в виде

$$
\left(\Delta_{\gamma}\right)_{x} u+\eta^{\frac{2 a}{a-1}} u_{\eta \eta}=0 .
$$

Интегральный оператор дробного порядка $\alpha>0$ следующего вида

$$
\left(\mathbf{U}_{\gamma}^{\alpha} \varphi\right)(x)=\int_{\mathbb{R}_{n}^{+}} \frac{\gamma \mathbf{T}_{x}^{y} \varphi(x)}{|y|^{n+|\gamma|-\alpha}} y^{\gamma} d y
$$

называется В-потенциалом Рисса (см. [4]). В [5] построено его обращение в виде B-гиперсингулярного интеграла. При $0<\alpha<1 B$-гиперсингулярный интеграл имеет вид

$$
C_{n, \gamma, a} \int_{\mathbb{R}_{+}^{n}} \frac{f(\xi)-\gamma \mathbf{T}_{x}^{\xi} f(x)}{|x|^{n+|\gamma|-a+1}} \xi^{\gamma} d \xi=\left(\mathbf{D}_{\gamma}^{1-a} f\right)(x)
$$

где $C_{n, \gamma, a}=\frac{1}{C(n, \gamma) S_{n, l}(\alpha)}, C(N, \gamma)=\frac{\Gamma\left(\frac{N+\alpha}{2}\right) \prod_{i=1}^{n} \Gamma\left(\frac{\gamma_{i}+1}{2}\right)}{2^{n} \Gamma\left(\frac{N+|\gamma|+\alpha}{2}\right) \pi^{n / 2}}, S_{N, l}(\alpha)=\beta_{N}(\alpha) \frac{A_{l}(\alpha)}{\sin \frac{\alpha \pi}{2}}$,

Лемма 5.1. Пусть $x \in \mathbb{R}_{+}^{n}, n>1 u$

$$
P(x, y)=C_{n, \gamma, a} \frac{y^{1-a}}{\left(|x|^{2}+y^{2}\right)^{\frac{n+|y|-a+1}{2}}}
$$

то для $|x|>\varepsilon \forall \varepsilon>0$ имеем

$$
\Delta_{\gamma, a} P(x, y)=0
$$

Доказательство. Действительно, непосредственные вычисления дают

$$
\begin{aligned}
& \Delta_{\gamma, a} P(x, y)=C_{n, \gamma, a} \Delta_{\gamma, a} \frac{y^{1-a}}{\left(|x|^{2}+y^{2}\right)^{\frac{n+|\gamma|-a+1}{2}}}= \\
& =C_{n, \gamma, a}\left(\sum_{i=1}^{n}\left(B_{\gamma_{i}}\right)_{x_{i}} \frac{y^{1-a}}{\left(|x|^{2}+y^{2}\right)^{\frac{n+|\gamma|-a+1}{2}}}+\left(B_{a}\right)_{y} \frac{y^{1-a}}{\left(|x|^{2}+y^{2}\right)^{\frac{n+|\gamma|-a+1}{2}}}\right)= \\
& =C_{n, \gamma, a}\left(y^{1-a}(a-|\gamma|-n-1) \sum_{i=1}^{n}\left(\left(\gamma_{i}+1\right)\left(|x|^{2}+y^{2}\right)^{\frac{a-|\gamma|-n-3}{2}}+x_{i}^{2}(a-n-|\gamma|-3)\left(|x|^{2}+y^{2}\right)^{\frac{a-|\gamma|-n-5}{2}}\right)-\right. \\
& \left.-y^{1-a}(a-|\gamma|-n-1)\left(|x|^{2}+y^{2}\right)^{\frac{a-|\gamma|-n-5}{2}}\left((a-3)|x|^{2}+y^{2}(|\gamma|+n)\right)\right)= \\
& =C_{n, \gamma, a}\left(y^{1-a}(a-|\gamma|-n-1)\left(|x|^{2}+y^{2}\right)^{\frac{a-|\gamma|-n-5}{2}} \sum_{i=1}^{n}\left(\left(\gamma_{i}+1\right)\left(|x|^{2}+y^{2}\right)+x_{i}^{2}(a-n-|\gamma|-3)\right)-\right. \\
& \left.-y^{1-a}(a-|\gamma|-n-1)\left(|x|^{2}+y^{2}\right)^{\frac{a-|\gamma|-n-5}{2}}\left((a-3)|x|^{2}+y^{2}(|\gamma|+n)\right)\right)= \\
& =C_{n, \gamma, a}\left(y^{1-a}(a-|\gamma|-n-1)\left(|x|^{2}+y^{2}\right)^{\frac{a-|\gamma|-n-5}{2}}\left((n+|\gamma|)\left(|x|^{2}+y^{2}\right)+|x|^{2}(a-n-|\gamma|-3)\right)+\right. \\
& \left.-y^{1-a}(a-|\gamma|-n-1)\left(|x|^{2}+y^{2}\right)^{\frac{a-|\gamma|-n-5}{2}}\left((a-3)|x|^{2}+y^{2}(|\gamma|+n)\right)\right)= \\
& =C_{n, \gamma, a}\left(y^{1-a}(a-|\gamma|-n-1)\left(|x|^{2}+y^{2}\right)^{\frac{a-|\gamma|-n-5}{2}}\left((n+|\gamma|) y^{2}+(a-3)|x|^{2}\right)+\right. \\
& \left.-y^{1-a}(a-|\gamma|-n-1)\left(|x|^{2}+y^{2}\right)^{\frac{a-|\gamma|-n-5}{2}}\left((a-3)|x|^{2}+y^{2}(|\gamma|+n)\right)\right)=0 \text {. }
\end{aligned}
$$


Следствие 5.1. Функция

$$
P(x, z)=C_{n, \gamma, a} \frac{\eta}{\left(|x|^{2}+(1-a)^{2} \eta^{\frac{2}{1-a}}\right)^{\frac{n+|\gamma|-a+1}{2}}}
$$

является решением уравнения (18).

Теорема 5.1. Пусть $x \in \mathbb{R}_{n}^{+}, n>1 u$

$$
P(x, y)=C_{n, \gamma, a} \frac{y^{1-a}}{\left(|x|^{2}+y^{2}\right)^{\frac{n+|\gamma|-a+1}{2}}}
$$

тогда $B_{\gamma_{j}} P(x, y) \in L_{\text {loc, } \gamma}^{1}\left(\mathbb{R}_{+}^{n}\right)$ и решение задачи (16)-(17) имеет вид

$$
u(x, y)=\int_{\mathbb{R}_{+}^{n}}{ }^{\gamma} \mathbf{T}_{x}^{\xi} P(x, y) f(\xi) \xi^{\gamma} d \xi
$$

Доказательство. Очевидно, что если $P(x, y)$ есть решение уравнения (16), то и $\int_{\mathbb{R}_{+}^{n}}{ }^{\gamma} \mathbf{T}_{x}^{\xi} P(x, y) f(\xi) \xi^{\gamma} d \xi$ есть решение (16). Кроме того,

$$
P(x, y)=y^{-n-|\gamma|} P(x / y, 1)
$$

и, следовательно,

$$
\lim _{y \rightarrow 0} P(x, y)=\delta_{\gamma}
$$

Тогда

$$
\lim _{y \rightarrow 0} \int_{\mathbb{R}_{+}^{n}}{ }^{\gamma} \mathbf{T}_{x}^{\xi} P(x, y) f(\xi) \xi^{\gamma} d \xi=\int_{\mathbb{R}_{+}^{n}}{ }^{\gamma} \mathbf{T}_{x}^{\xi} \delta_{\gamma}(x) f(\xi) \xi^{\gamma} d \xi=f(x) .
$$

Что и завершает доказательство.

Следствие 5.2. Решение задачи (18)-(17) имеет вид $u(x, z)=\int_{\mathbb{R}_{+}^{n}}{ }^{\gamma} \mathbf{T}_{x}^{\xi} P(x, z) f(\xi) \xi^{\gamma} d \xi$.

Теорема 5.2. Пусть $f \in L_{p}^{\gamma}\left(\mathbb{R}_{+}^{n}\right), 0<a<1, s=\frac{1-a}{2}, s<\frac{n+|\gamma|}{p}, \eta=\left(\frac{y}{1-a}\right)^{1-a}$

$$
\left(-\Delta_{\gamma}\right)^{s} f(x)=-\lim _{y \rightarrow+0} y^{a} u_{y}(x, y)=u_{\eta}(x, 0)
$$

Доказательство. Имеем

$$
\begin{gathered}
u_{\eta}(x, 0)=\lim _{\eta \rightarrow 0} \frac{u(x, \eta)-u(x, 0)}{\eta}=\lim _{z \rightarrow 0} \frac{1}{\eta} \int_{\mathbb{R}_{+}^{n}}{ }^{\gamma} \mathbf{T}_{x}^{\xi} P(x, \eta)(f(\xi)-f(x)) \xi^{\gamma} d \xi= \\
=C_{n, \gamma, a} \lim _{\eta \rightarrow 0} \int_{\mathbb{R}_{+}^{n}}{ }^{\gamma} \mathbf{T}_{x}^{\xi}\left(\left(|x|^{2}+(1-a)^{2} \eta^{\frac{2}{1-a}}\right)^{\frac{a-n-|\gamma|-1}{2}}\right)(f(\xi)-f(x)) \xi^{\gamma} d \xi= \\
=C_{n, \gamma, a} \int_{\mathbb{R}_{+}^{n}} \frac{f(\xi)-{ }^{\gamma} \mathbf{T}_{x}^{\xi} f(x)}{|x|^{n+|\gamma|-a+1}} \xi^{\gamma} d \xi=\left(\mathbf{D}_{\gamma}^{1-a} f\right)(x),
\end{gathered}
$$

где $\mathbf{D}_{\gamma}^{1-a}=\left(-\Delta_{\gamma}\right)^{\frac{1-a}{2}}-B-$ гиперсингулярный интеграл (см. [4, 5]).

\section{Список литературы}

1. Житомирский Я. И. 1955. Задача Коши для систем линейных уравнений в частных производных с дифференциальными операторами типа Бесселя. Матем. сб., 36(78)(2): 299-310.

2. Киприянов И. А. 1997. Сингулярные эллиптические краевые задачи. М., Наука-Физматлит, 204.

3. Левитан Б. М. 1951. Разложения по функциям Бесселя в ряды и интегралы Фурье. М., УМН. 6:2(42): $102-143$.

4. Ляхов Л. Н. 1991. Обращение В-потенциалов Рисса. Докл. АН СССР, 321(3): 466-469.

5. Ляхов Л. Н. 1990. Об одном классе гиперсингулярных интегралов. Докл. АН СССР, 315(2): 291-296. 
6. Хермандер Л. 1986. Анализ линейных дифференциальных операторов с частными производными. 1. Теория распределений. М., Мир, 482.

7. Шишкина Э. Л. 2019. Обобщенная дивергентная теорема и второе тождество Грина для $B$-эллиптических и $B$-гиперболических операторов. Научные ведомости Белгородского государственного университета. Серия: Математика. Физика. 51(4): 506-513.

\section{References}

1. Zhitomirskii Ya. I. 1955. Cauchy's problem for systems of linear partial differential equations with differential operators of Bessel type. Mat. Sb. (N.S.), 36(78)(2): 299-310 (in Russian).

2. Kipriyanov I. A. 1997. Singular Elliptic Boundary Value Problems. M.: Nauka-Fizmtlit, 204 (in Russian).

3. Levitan B. M. 1951. Expansion in Fourier series and integrals with Bessel functions. Uspekhi Mat. Nauk, 6:2(42): 102-143 (in Russian).

4. Lyakhov L. N. 1992. Inversion of Riesz B-potentials. Dokl. Math., 44(3): 717-720(in Russian).

5. Lyakhov L. N. 1991. A class of hypersingular integrals. Dokl. Math., 42(3): 765-769 (in Russian).

6. Hermander L. 1986. Analysis of linear partial differential operators. 1. The theory of distributions. M ., Mir, 482 (in Russian).

7. Shishkina E. L. 2019. Generalized divergent theorem and second Green identity of $B$-elliptic and $B$-hyperbolic operators. Belgorod State University Scientific Bulletin. Mathematics. Physics. 51(4): 506-513 (in Russian).

Конфликт интересов: о потенциальном конфликте интересов не сообщалось.

Conflict of interest: no potential conflict of interest related to this article was reported.

Получена 08.12.2021

\section{СВЕДЕНИЯ ОБ АВТОРАХ}

Алзамили Хитам - аспирант Белгородского государственного национального исследовательского университето (НИУ «БелГУ»)

http://orcid.org/0000-0003-1354-6478

ул. Победы, 85, Белгород, 308015, Россия

E-mail: alzamili.khitam@mail.ru

Шишкина Элина Леонидовна - доктор физико-математических наук, доцент, профессор Воронежского Государственного Университета и Белгородского государственного национального исследовательского университета (НИУ «БелГУ»)

http://orcid.org/0000-0003-4083-1207

Университетская пл., д.1, Воронеж, 394018, Россия; ул. Победы, 85, Белгород, 308015, Россия

E-mail: ilina_dico@mail.ru

\section{INFORMATION ABOUT THE AUTHORS}

Khitam Alzamili - Postgraduate student, Belgorod State National Research University (NRU "BelGU"), Belgorod, Russia

Elina Shishkina - Doctor of Sciences Phys. Math., Associate Professor, Professor of Voronezh State University and Belgorod State National Research University (NRU "BelGU"), Voronezh, Belgorod, Russia 